\title{
A Service Oriented and Agent-Based Architecture for the e-Collaboration of SMEs
}

\author{
Ioannis Ignatiadis ${ }^{1}$, Dimitrios Tektonidis ${ }^{2}$, Adomas Svirskas ${ }^{1,3}$, \\ Jonathan Briggs ${ }^{1}$, Stamatia-Ann Katriou ${ }^{2}$, Adamantios Koumpis ${ }^{2}$ \\ ${ }^{1}$ Kingston University, Faculty of Computing, Information Systems and \\ Mathematics, Penrhyn Road, SURREY KT1 2EE, UK \\ ${ }^{2}$ ALTEC S.A. Research Programs Division, M. Kalou 6, Thessaloniki 546 29, \\ GREECE \\ ${ }^{3}$ Institut Eurécom, 2229 Route des Crêtes, Sophia-Antipolis 06560, FRANCE
}

\begin{abstract}
The concept of a Service-Oriented Architecture (SOA) to flexibly address business needs with the use of Information Technology (IT) is increasingly being recognized as important for a company's agility and responsiveness to change. A SOA approach can also help to design more agile web portals, in order to enable companies to increase their responsiveness and adaptability with regards to addressing a business opportunity as a collaborative Virtual Organization (VO). Value can be added to the operations of such a VO with the use of Intelligent Agents to automate the processes of finding collaboration partners and negotiating the creation of VOs based on user-defined business rules. This paper discusses these concepts and their benefits for Virtual Organizations based on the work of a European Union (EU) co-funded Information Society Technologies (IST) project examining these issues for Small and Medium Enterprises (SMEs) in the European Enterprise Resource Planning (ERP) industry.
\end{abstract}

\section{Introduction}

Small and Medium-size Enterprises (SMEs) participating in today's global market are often ill-prepared in competing with their larger counterparts, who have established international activities with customers, suppliers and partners. The problems that

Please use the following format when citing this chapter:

Ignatiadis, I., Tektonidis, D., Svirskas, A., Briggs, J., Katriou, S. -A., Koumpis, A., 2008, in IFIP International Federation for Information Processing, Volume 286; Towards Sustainable Society on Ubiquitous Networks, eds. Oya, M., Uda, R., Yasunobu, C., (Boston: Springer), pp. 125-137. 
SMEs in particular face have to do with limited partner networks in other countries, their narrow focus on specific geographic areas, and the limited amount of skills and resources to enable them to expand their activities and compete globally.

One way to overcome these limitations is to form a collaborative Virtual Organization (VO) with other companies facing the same problems but having complementary skills and resources. Such VOs are formed in response to more intensive competition, more specialized markets, faster technological change and shorter product (or service) life cycles (Aldrich 1999, Davidow and Malone 1992, Hagel and Armstrong 1997). The advantages for SMEs in forming and participating in such VOs include reduced operational and partner search costs, increased operational efficiency, increase of competitiveness against large corporations, and better meeting end customer needs by the sharing of knowledge and competencies among VO participants (Rautenstrauch 2002).

To support the formation and sustainability of online collaborations, a business model supported by an online collaboration platform must be implemented. In this paper we argue for the importance of using Service-Oriented Architecture (SOA) principles for the building of such platforms, as well as using intelligent software agents to automate some of the operations of the collaborative VOs. Our background is the European Union (EU) co-funded project Panda (PANDA-Project 2006), where we examine the applicability of SOA and Intelligent Agents in the context of the creation and maintenance of e-collaborations in the European Enterprise Resource Planning (ERP) industry of SMEs.

In the sections that follow we first present background literature on the concepts of SOA and Intelligent Agents, followed by the description of the Panda project where these concepts are examined. We then present the SOA and Intelligent Agent-based architecture in the Panda project, followed by the discussion of its benefits. The paper is concluded with the current state of developments and future research in the Panda project.

\section{Service-Oriented Architectures and Intelligent Agents}

\subsection{Service-Oriented Architectures}

Service-Oriented Architecture (SOA) is an architectural style in which software applications are organized as a set of loosely coupled services (Harding 2007). The SOA paradigm enables to link business and computational resources (mainly organizations, applications and data) on demand. It is seen to be essential for delivering business agility and IT flexibility. It also facilitates the alignment of existing IT infrastructures to achieve (internal or external) enterprise connectivity, by removing redundancies, generating unified collaboration tools, and streamlining IT processes. The benefits from this approach are an increase in business agility to respond to future changes by adapting and reorganizing the existing services, as well 
as creating new services to fit in the existing IT architecture, in order to address changed business requirements. This applies to both internal operations, as well as external ones (e.g. providing new services to customers or changing the way the interactions with suppliers are carried out). A well-executed SOA implementation can bridge the gap between enterprise architecture and business strategy, with companies achieving a closer alignment of IT and the business, while in parallel implementing the robust reuse of existing technology and application code with agility and cost effectiveness (Laurent 2007).

Traditional systems were built as monoliths, making change complex, timeconsuming and costly. The tight interconnection of such systems meant that even small changes could ripple across the applications and cause major rewriting (Tews 2007). The approach that SOA takes is that instead of tightly integrated application code, applications are built using loosely-coupled modules called "services", with each service describing an interface that can be accessed regardless of programming language, and without the need to access or understand the underlying code. Such services can be provided within enterprise applications, as interfaces to legacy systems, as new development, or from external providers in industry and government. From a business point of view, the goals are quicker time to market with innovative offerings, greater productivity, and reduced integration costs.

A SOA architecture is based on six assumptions (Brandl 2007): applications are loosely coupled; interface transactions are stateless; interface follows the RPC (remote procedure call) model; interface is message-based; messages use XML (eXtensible Markup Language) data; and interfaces may support both synchronous and asynchronous transactions. Viewed as an approach to building IT systems to match flexible business requirements, SOA connects applications across a network via a common communications protocol, allowing organizations to reuse old and future software, often with the help of Web Services (Brodkin 2007). SOA incorporates standards like SOAP (Simple Object Access Protocol) and XML, to deliver standard messaging formats and increased reuse of information assets at lower integration costs (Larrivee 2007).

\subsection{Intelligent Agents}

Service-Oriented Architectures do not provide any support for rational (semi-) automated selections when it comes to the creation of Virtual Organizations. Such rational selection is crucial for establishing and operating viable business formations. This issue can be addressed by enriching SOA with Intelligent Agents. Interests of individual VO members are represented by Intelligent Agents, acting according to customizable business rules. Although previous research on the role of Intelligent Agents in Virtual Organization has been carried out (e.g. Andrade et al. 2005, Guidi-Polanco et al. 2005, Oliveira and Rocha 2000, Petersen 2003, 2007, Svirskas et al. 2006), in this paper we are mostly concerned with the framing of Intelligent Agents within an overall SOA architecture, and the expected benefits of such an approach.

Practically this means exposing results of agent operations via standard Web Services interfaces, enabling easy integration of these results into ad-hoc mash-ups 
and portals. Maximilien and Singh (2005) distinguish between two primary classifications of consumer-to-provider interaction styles in services: simple interactions and agent-mediated interactions. Simple interactions occur directly between a service consumer and a service provider. Agent-mediated interactions use an intermediary agent that facilitates some aspects of the interaction. In our work we are mostly concerned with agent-mediated interaction styles as they allow providing richer solutions.

One also needs to make sure that these two types of entities (agents and services) coexist peacefully within a single architecture and interoperate properly. Maximilien \& Singh propose a framework that augments a typical Service-Oriented Architecture with agents. Their principal idea is to install software agents between service consumers and each service that they consume. These consumer service agents expose the same interface as the service. However, they augment the service interface with agent-specific methods. The consumer communicates its needs via the augmented agent interface. Service method invocations are done via the service agent who, in turn, monitors and forwards all calls to the selected service.

The concept of multiple agents can also be useful in general-purpose Web Service composition. Maamar et al. (2005) present an agent-based and context-oriented approach that supports the composition of Web Services. To reduce the complexity featuring the composition of Web Services two concepts are put forward in their work, namely, software agent and context. During the composition process, software agents engage in conversations with their peers to agree on the Web Services that participate in this process. Conversations between agents take into account the execution context of the Web Services. The security of the computing resources on which the Web Services are executed constitutes another core component of the agent-based and context-oriented approach presented by Maamar et al.

The benefits of combining SOA and Intelligent Agents are foreseen to be increased adaptability and flexibility to organizational needs regarding VO processes. However, there is still much research to be done on the (business and technical) aspects of the combination of SOA and Intelligent Agents in the context of VOs. In addition, the standards associated with SOA (e.g. Web Services standards) are still to become mature and established. SOA may also not be particularly applicable in mission or safety-critical applications (Bloomberg and Schmelzer 2006). However, the use of these concepts in the Panda project, the associated architecture and the expected benefits are presented in the following sections.

\section{The Panda Project}

Panda (PANDA-Project 2006). examines e-collaboration in the European ERP industry of SMEs, facilitated with the formation and management of Virtual Organizations. Currently, the European ERP market (which is a major part of ICT business applications market in Europe - worth approximately $€ 15$ billion) is dominated by large multinational actors, most of them outside the EU, focusing on high-end market segments (ERP solutions for large multinational companies). However, as the high-end 
market has reached maturity, there is a shift of interest towards small and medium market segments (ERP solutions for small companies and SMEs).

Recognizing this potential for ERP value chains of SMEs, Panda aims to provide a powerful framework of e-business services, dedicated to addressing current inefficiencies in the operations of such value chains, as well as facilitating international ecollaborations based on local actors and alliances. Panda proposes the development of a new partnership based e-business model for SMEs such as ERP vendors, their national representatives, dealers and consultants. Panda also includes the development of a set of integrated supporting technologies in the form of a platform (portal) that will engage and involve local players in flexible multinational e-collaborations. These collaborations concern the provision of services towards an end customer (e.g. ERP implementations, upgrades, customizations, training, support, etc) which require the expertise of more than one partner.

The pilot installation of the prototype platform takes advantage of Panda's two large international 'informal' ERP value chains, comprising 2 ERP vendors (SMEs), 200 ERP national representatives and dealers and 50 IT consultants, who are active in 7 European countries (Greece, Romania, Bulgaria, Germany, Hungary, Finland and Sweden). The two value chains examined practically represent two different edges that appear in the ERP industry: (a) rigid - hierarchical value chain supporting a fully protected and licensed ERP product, (b) loose virtual community / value network of collaborating actors supporting an open source ERP solution.

The main functional requirements that arose from the users' perspective in Panda were the need to identify online suitable collaboration partners for participation in ERP projects, the efficient communication of the project's requirements and status, experience sharing from previous similar ERP projects, and standardization of contracts for international collaborations. Apart from the functional requirements however, other non-functional requirements which emerged as important were the flexibility of platform operations to match different value chain characteristics, while also allowing interoperability between platform architecture and partners' own systems. In addition, a degree of automation of operations carried out in the Panda platform as part of VO establishment was also deemed important. As such, the concepts of SOA and Intelligent Agents needed to be considered in the design of the architecture of the Panda platform. This architecture and its benefits are presented next.

\section{E-Collaboration Architecture and Operations}

\subsection{System Architecture}

Figure 1 illustrates Panda's SOA architecture (implemented with .NET Web Services) - the central point of user interaction is a portal (based on Microsoft Web Part's technology), accessed by human users and software agents. Interests of individual members of the Panda ecosystem are represented by Intelligent Agents (using FIPA 
standards and written in Java), acting according to customizable business rules (implemented using JBoss).

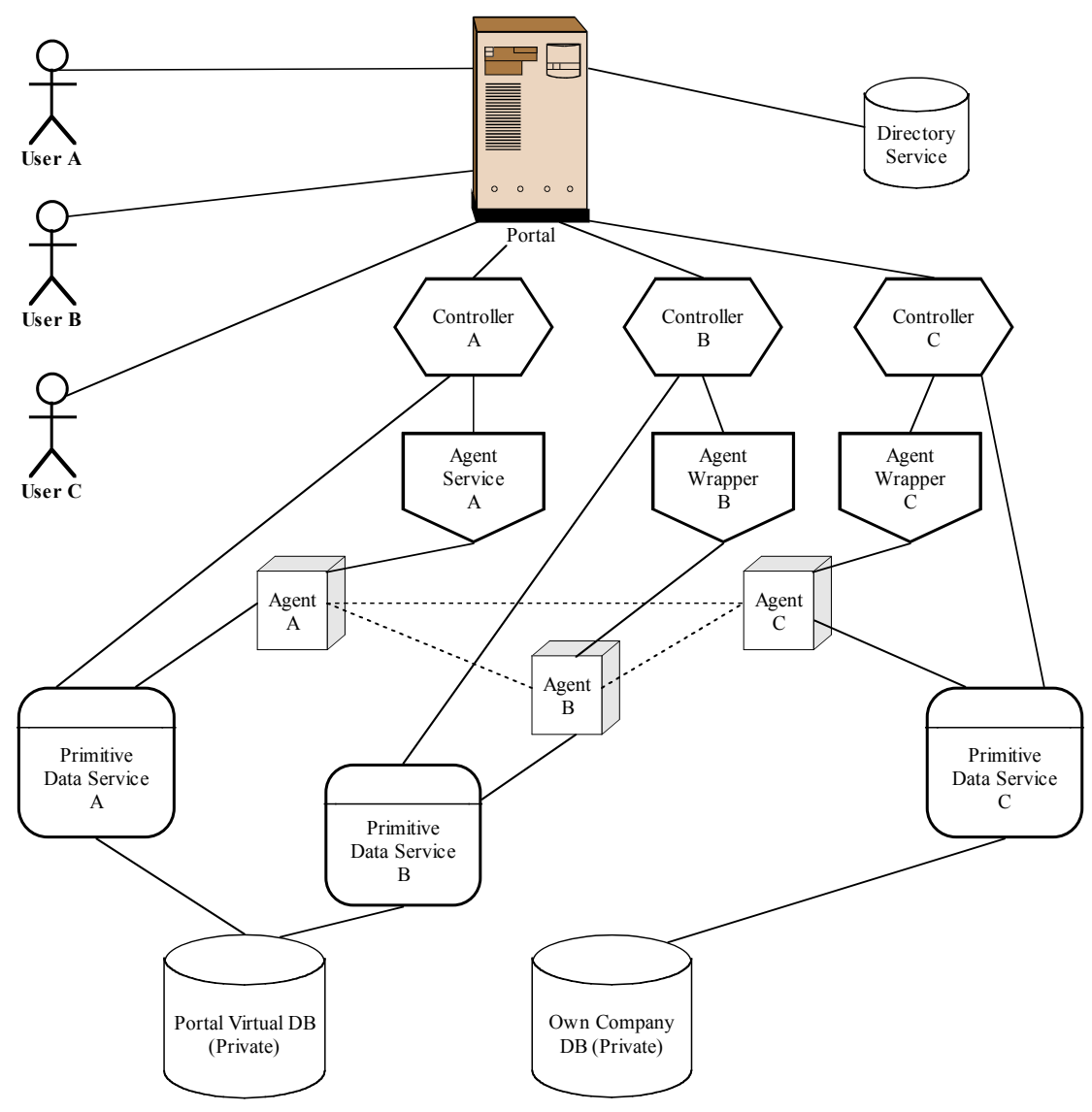

Fig. 1. Panda's SOA and agent-based architecture

The components that comprise the Panda platform are the following:

- Directory Service: A common repository containing login information, standard values like ERP modules, languages, industry sectors etc. The directory service can be accessed by a Web Service.

- Controller: User functionality in the portal that enables the creation and submission of requests to the Intelligent Agent.

- Agent Service: A Web Service that enables the submission of a Collaboration Request to the Agent and the acquisition of the list of the proposed VOs. This can also be implemented by a Web Service wrapper around agent functionality.

- Agent: The company's Intelligent Agent that undertakes the negotiations and the formation of the VOs. Agents communicate with each other to exchange information and engage in negotiations. 
- Primitive Data Services: Set of simple Web Services that enable Agents and Controller to access the company's private repository (company database).

- Private repository (Company database): The repository that contains private information of the company like Company Profile, Collaboration Requests, Contracts etc. This can be a dedicated (virtual) storage within the portal, or can be at the company's legacy systems.

\subsection{Agent Operations}

The Panda agent system is composed of independent distributed instances of Partner Agent interacting with each other and with a set of centralized platform services. The main purpose of agents is to identify and negotiate with potential partners for collaboration in a VO, and suggest a number of such potential VOs based on various criteria such as price, deadlines of tasks, previous experiences and reputation of participants, etc. As such, the scope of agent contribution in Panda can be formulated as agentbased negotiation towards VO formation using dynamic (semi-)private knowledge. The agents consequently represent individual (distributed) actors participating in VO formation processes taking into account (i) individual private constrains and preferences (e.g. with regards to types of projects sought, availabilities of staff, pricing rules, etc) that are hidden to other actors, and (ii) minimization of disclosure of such private information.

Agents get active in the system once a Collaboration Request (CR) for the identification of suitable partners for collaborating in an ERP project is submitted in the system by a human user. Once a Collaboration Request (CR) has been created by a company in Panda and sent to potential partners via the corresponding agent, the companies that receive the request may begin negotiations by editing the request and sending a proposal back to the company via their agents. Further negotiations after the first response from the human users can be subsequently carried out automatically by agents (e.g. in terms of prices or deadlines). At the end of the negotiations the agent of the Collaboration Request issuer can create VO proposals that can be seen by the human user and one of these proposals potentially selected. The user who made the response proposal is then informed for his/her selection in a VO and the human requester of the collaboration can start composing a contract with his/her partner(s). Once the contract has been defined and agreed upon by all involved parties the project is then started. Project management activities can be carried out with an appropriate tool connected via Web Services to the Panda portal. At the end of the operations of the VO its results can be archived, and VO partners can rate each other with regards to their collaboration efficiency.

As has been mentioned, agents are involved in negotiations about CR details that lead to VO formation. Each agent is dedicated to a single company - the member of the value chain. The interaction amongst agents in Panda for the handling of Collaboration Requests (with human advisement) and VO proposals is shown in Figure 2 below.

In their negotiations, agents can be guided by user-defined business rules. Agents need to decide which of these rules are applicable in each type of negotiation, and 
need to flexibly adapt and prioritize those rules as the negotiations progress, potentially also guided by human input to correct decisions taken. Such rules can be set by each user to guide his/her agent, and can be implemented using a rules engine. Indicative user-defined business rules for the negotiation amongst agents can include for example:

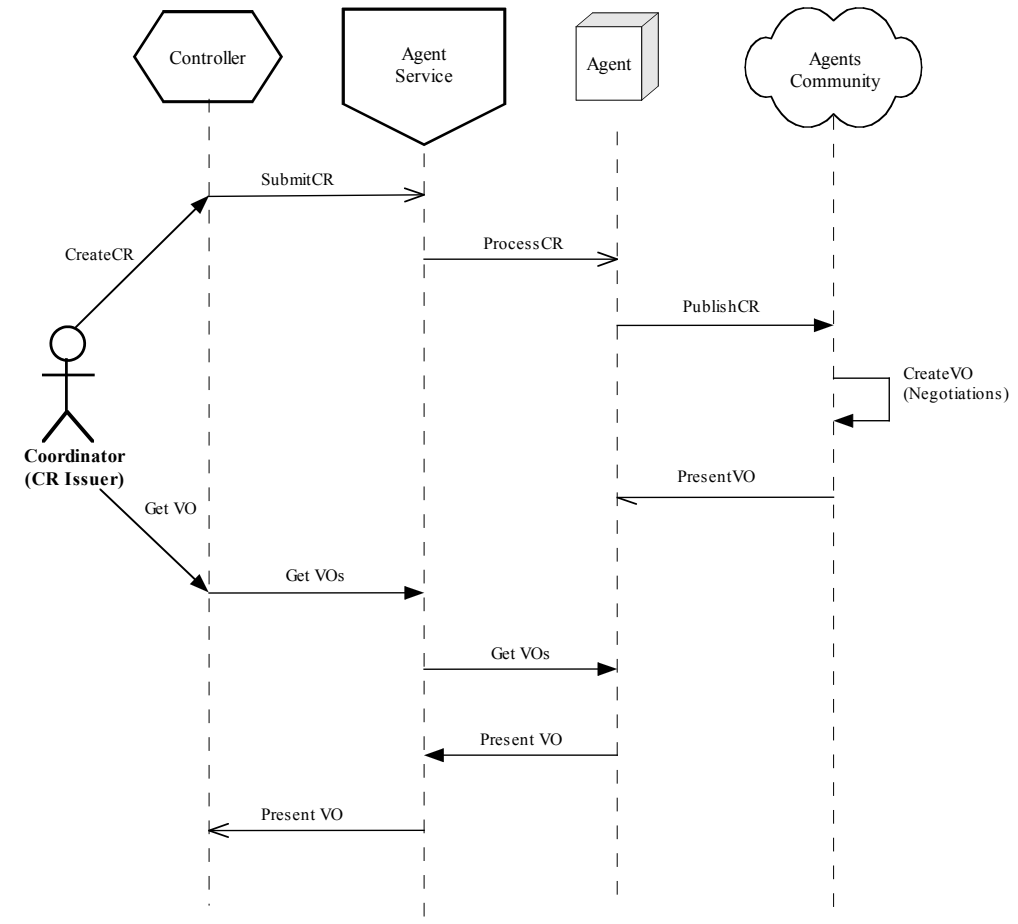

Fig. 2. Agent-based Collaboration Request (CR) processing and Virtual Organization (VO) handling

- My tolerance on price is $-\mathrm{x} \%$ to $+\mathrm{y} \%$.

- I am prepared to wait $\mathrm{x}$ days to get a better price.

- I will pay more to finish the project sooner.

- Only consider partners in the same region/country as me.

- I do not want to deal with partners in my blacklist.

- I do not want to participate in projects less than x Euros.

These rules are represented in a JBoss rules engine, and agents need to decide the priority of each of these rules and resolve potential conflicts amongst the rules. As the negotiation amongst the agents is mainly based on price (and to a lesser degree deadlines of tasks), the majority of the business rules define strategies for negotiating the pricing arrangements amongst the partners. 


\subsection{SOA and Agent Benefits in Panda}

The importance of adopting a Service-Oriented Architecture to increase an organization's agility and flexibility is becoming clearer for many organizations interested in gaining a competitive advantage by differentiating their services and becoming better prepared to respond to changes in their market (Bloomberg and Schmelzer 2006). Extending the SOA principles to a VO setting in the Panda project, it is argued that the SOA advantages can also be obtained for a conglomeration of companies coming together to synergistically exploit complementary skills and competencies. Although there is not a shortage of platforms for firms to collaborate online in particular industries or across industries, the advantages of adopting a SOA approach for such platforms offered by the adaptability and easy reconfigurability to match heterogeneous VOs' business requirements, need to be taken into consideration. The benefits of using a SOA approach in Panda in particular is the reusability, interoperability and adaptation of the various Panda components to different value chains (e.g. with regards to how reputation or project management are handled), as well as companies' operational characteristics (e.g. with regards to how company profiles and company private data are handled). Different enterprises connecting to the Panda platform can exploit Web Service connectivity to pass their data (e.g. profiles, pricelists, etc) to and from the portal, as well as using data from the portal, implementing a "data as a service" layer. Services provided by the Panda platform can also be substituted by alternative ones, or new ones can be written, to ensure compatibility with different ERP value chain requirements, in addition for allowing for the possibility for the Panda platform to be exploited outside the ERP industry, implementing a "software as a service" layer. The SOA conceptual approach in Panda is depicted in Figure 3 below.

In contrast to costly and timely hardwiring of point-to-point connections between companies in order to promote interoperability and collaboration, a distributed SOA approach adopted in Panda enables companies to more flexibly participate in a platform that can consider the technical (infrastructure) and business (operational) capabilities of enterprises to enable them to efficiently engage in collaborative activities with other partners. 


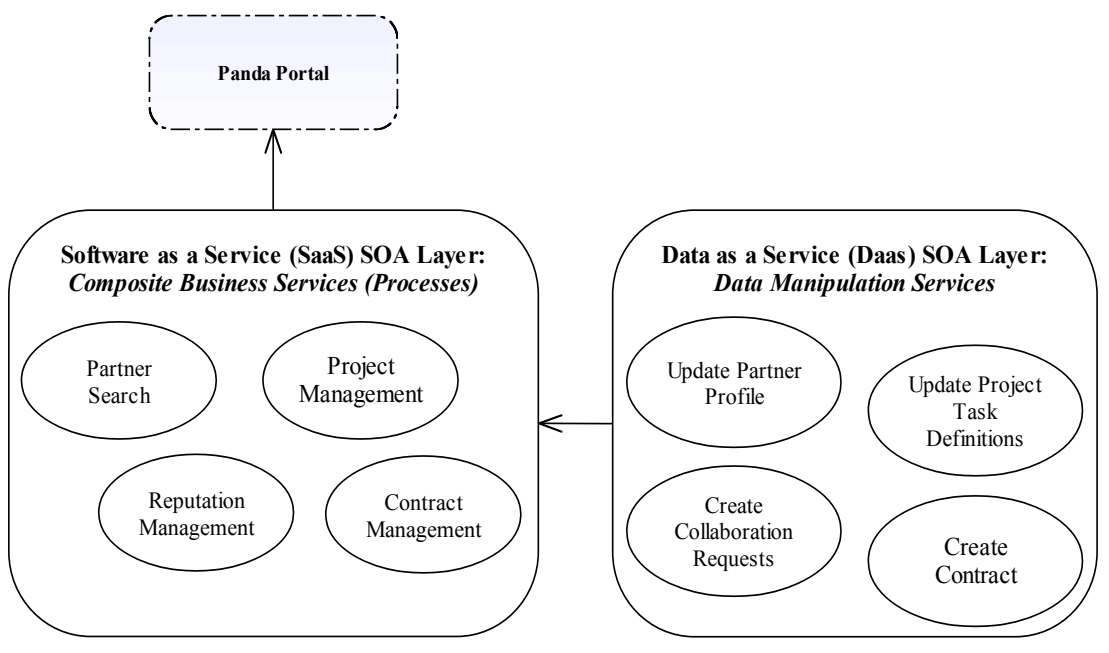

Fig. 3. SOA conceptual approach in Panda

In addition, in contrast to large ERP vendors (such as SAP, Oracle) having their own business ecosystems centered around the provision, installation, maintenance and support of their products, for SMEs in the ERP industry the participation in a flexible, adaptable, and partially automated (with Intelligent Agents) e-collaboration platform can increase their synergies, expand their activities and lower their costs of operation, making them better suited to compete with their larger counterparts.

With regards to Intelligent Agents, as has been mentioned these play the role of the partners' representatives for (semi-)automated negotiation that support the e-business acceleration in the ERP value chain domain. One of the goals is to provide negotiation-based matchmaking methods that take into account (i) limited information provision, (ii) multi criteria evaluation of proposals, (iii) private preferences and metrics (in terms of different weights for criteria used to find potential collaborators) for each participant. Using (static) public data, previous experience, or reputation mechanism, the collaboration requester's agent can carry out negotiations with a selected set of potential partners that provide the highest probability to match the requester's preferences. The peer-to-peer negotiation is used for gathering semi-private knowledge to be able to evaluate the participants (proposals are based on participants' private preferences and availability and can't be evaluated without negotiation) and construct VOs. The finalization of the matchmaking is done by the VO proposals evaluation and there is a possible backtracking when the evaluation gives non-satisfactory results. This automation of partner finding and VO creation processes (supported by human advisement) is expected to give significant time and cost savings for the relevant SMEs in the ERP industry. 


\section{Conclusions}

The main concerns of Panda include: (1) taking prevailing best practices from SOA and associated issues (e.g. what can be provided as a service and in which level of abstraction, as well as interoperability at the data and software levels), and finding best ways to apply them to SMEs and their business needs regarding collaboration with other partners, (2) examining the degree to which processes within a VO can be automated with the use of Intelligent Agents.

It is expected that the combination of Intelligent Agents aiding in the automation of processes (such as negotiation-based matchmaking for the formation of VOs), together with the adoption of a SOA approach promoting flexibility, interoperability and adaptability to user needs, can contribute towards the building and maintenance of a community of member organizations with similar or complementary skills and competencies. At the time of writing of this paper a prototype system using SOA and Intelligent Agent principles was completed, and given to a number of SMEs in the European ERP industry for testing and practical evaluation of the expected benefits of such an approach. In addition, the issues of building security and trust in online environments (Dimitrakos et al. 2004, Ignatiadis et al. 2006, Msanjila and Afsarmanesh 2007) in order to increase collaboration, as well as adopting efficient and effective SOA governance (Bloomberg and Schmelzer 2006, Larrivee 2007, Laurent 2007), will also be examined in future stages of the project, together with the development of its business plan.

Although the Panda project is exemplified in the ERP industry, the concepts, business and developed technological aspects could also be applied in other settings where collaborative projects are implemented. This includes practically any business sector where business-oriented software solutions (i.e. software products coupled with value added services to form 'extended' solutions) are used. The applicability of Panda in these areas is also a matter of future research, together with examining Web 2.0 principles to increase collaboration amongst partners.

\section{Acknowledgements}

The authors of this paper wish to acknowledge the kind contribution of the European Commission towards funding of the Panda project.

\section{References}

Aldrich, D. F. (1999). Mastering The Digital Marketplace. Chichester: John Wiley.

Andrade, F., Neves, J., Novais, P., Machado, J., Abelha, A. (2005). Legal Security and Credibility in Agent Based Virtual Enterprises. Paper presented at the PRO-VE'05 - 6th IFIP Working Conference on Virtual Enterprises, Valencia, Spain, 26 - 28 September. 
136 Ioannis Ignatiadis, Dimitrios Tektonidis, Adomas Svirskas, Jonathan Briggs, StamatiaAnn Katriou, Adamantios Koumpis

Bloomberg, J., Schmelzer, R. (2006). Service Orient or Be Doomed!: How Service Orientation Will Change Your Business. Hoboken, New Jersey: Wiley.

Brandl, D. (2007). SOA explained. Control Engineering, 54(8), 22-22.

Brodkin, J. (2007). Are SOAs saving anyone money? Network World, 24(28), 24-25.

Davidow, W. H., Malone, S. M. (1992). The Virtual Corporation - Structuring And Revitalizing The Corporation For The 21st Century. New York: Harper Collins.

Dimitrakos, T., Wilson, M., Ristol, S. (2004). TrustCoM - A Trust and Contract Management Framework enabling Secure Collaborations in Dynamic Virtual Organisations. Sophia Antipolis, France.

Guidi-Polanco, F., Cubillos, C., Menga, G. (2005). The Global Automation Platform: An Agentbased Framework for Virtual Organizations. Paper presented at the PRO-VE'05 - 6th IFIP Working Conference on Virtual Enterprises, Valencia, Spain, 26 - 28 September.

Hagel, J., Armstrong, A. (1997). Net.Gain: Expanding Markets Through Virtual Communities. Harvard: Harvard Business School Press.

Harding, C. (2007). An Open Marketplace for Services. DM Review, 17(9), 20-39.

Ignatiadis, I., Svirskas, A., Roberts, B., Tarabanis, K. (2006). Promoting Trust in B2B Virtual Organizations through Business and Technological Infrastructures. International Journal of Networking and Virtual Organizations, Special Issue on: Trust Development and Management in Virtual Organizations, 3(4), 395-411.

Larrivee, B. (2007). SOA: No Governance Needed. Or Is It? AIIM E-DOC, 21(5), 24-25.

Laurent, W. (2007). The Importance of SOA Governance. DM Review, 17(8), 38-38.

Maamar, Z., Mostefaoui, S. K., Yahyaoui, H. (2005). Toward an Agent-Based and Context-Oriented Approach for Web Services Composition. IEEE Transactions on Knowledge and Data Engineering, $17(5), 686-697$.

Maximilien, E. M., Singh, M. P. (2005). Toward web services interaction styles. Paper presented at the 2005 IEEE International Conference on Services Computing (SCC'05), Orlando, Florida, USA, 11-15 July.

Msanjila, S. S., Afsarmanesh, H. (2007). Towards Establishing Trust Relationships Among Organizations in VBEs. Paper presented at the PRO-VE'07: 8th IFIP Working Conference on Virtual Enterprises, Guimaraes, Portugal, 10-12 September.

Oliveira, E., Rocha, A. P. (2000). Agents advanced features for negotiation in Electronic Commerce and Virtual Organisations formation process. In C. Sierra, F. Dignum (Eds.), European Perspectives on Agent Mediated Electronic Commerce: Springer-Verlag.

PANDA-Project. (2006). EU IST-027169. http://www.panda-project.com. Accessed April 2007.

Petersen, S. A. (2003). An Agent-based Evaluation Framework for Supporting Virtual Enterprise Formation. Paper presented at the Twelfth International Workshop on Enabling Technologies: Infrastructure for Collaborative Enterprises, Linz, Austria, 9-11 June.

Petersen, S. A. (2007). Virtual enterprise formation and partner selection: an analysis using case studies. International Journal of Networking and Virtual Organizations, 4(2), 201-215. 
Rautenstrauch, T. (2002). The Virtual Corporation: A Strategic Option For Small And Medium-Sized Enterprises (SMEs). Paper presented at the Association for Small Business and Entrepreneurship, St. Louis.

Svirskas, A., Ignatiadis, I., Roberts, B., Wilson, M. (2006). Virtual Organization Management using Web Service Choreography and Software Agents. Paper presented at the PRO-VE'06: 7th IFIP Working Conference on Virtual Enterprises, Helsinki, Finland.

Tews, R. (2007). Beyond IT: The business value of SOA. AIIM E-DOC, 21(5), 14-17. 